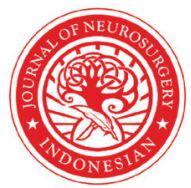

Indonesian Journal of Neurosurgery

\section{Relationship between CT scan density and hematoma age on chronic subdural hematoma cases}

\author{
Zulfadli Rizky Akbar ${ }^{1 *}$, Agus Turchan¹, Sri Andreani Utomo², Dyah Fauziah³
}

'Department of Neurosurgery, Faculty of Medicine Airlangga University/ Dr. Soetomo Academic General Hospital, Indonesia

${ }^{2}$ Department of Radiology, Faculty of Medicine Airlangga University/ Dr. Soetomo Academic General Hospital, Indonesia

${ }^{3}$ Department of Anatomical Pathology, Faculty of Medicine Airlangga University/ Dr. Soetomo Academic General Hospital, Indonesia

\section{*Corresponding to:}

Zulfadli Rizky Akbar; Department of Neurosurgery, Faculty of Medicine Airlangga University/ Dr. Soetomo Academic General Hospital, Indonesia; zulfadli.r.akbar@gmail.com;

\section{Received: 2020-06-03} Accepted: 2021-01-18 Published: 2021-04-22

\section{ABSTRACT}

Background: Subdural hematomas (SDH) can occur in one in three people with severe head injuries. Subdural hematomas are increasingly being found with age. Study of correlation of histopathological changes in hematoma and radiological contents of cases of subdural hematoma are still very limited.

Objective: In this research, SDH histopathological changes are expected to be a reference and consideration in the next invasive therapeutic action. This study also aims to look for other approaches in determining the therapeutic actions in acute subdural hematomas by comparing the clinical, radiological, and histopathological profiles of blood deposits from subdural hematomas

Methods: This study is a clinical observational study with a cross sectional study approach. Primary data were obtained from all subdural hematoma patients. CT Scan was performed and the results were read by Radiologists. Hematoma blood samples was analysed microscopically and morphologically by Pathologist at our hospital.

Results: The average value of CT Scan blood reading density of Chronic SDH patients in RSUD Dr. Soetomo which was operated on in 2018-2019 was 31.30 ( \pm 11.47 ) HU with the smallest value of 4.0 and the largest 54 HU. Average day of events was 36 \pm 36.66 days with the shortest occurrence day 12 days and the longest 150 days. There is an influence between the (T Scan reading density and the day of the incident. The calculation results obtained a negative correlation $(r=-0.814 ; p<0.001)$ between the CT Scan density value and the day of occurrence. Statistical calculation of the coefficient BO for the dependent variable (day of occurrence) is 147.557 and the coefficient of B1 for the HU value from the CT Scan reading is - 3.417 . In this study, the histology generally did not represent chronic SDH readings specifically.

Conclusion: There's a negative correlation between the number of incident days with the CT Scan density where the longer the event day the lower the CT Scan density value.

Keywords: Subdural Hematomas, CT Scan Density, Histopathological

Cite This Article: Akbar, Z.R., Turchan, A., Utomo, S.A., Fauziah, D. 2021. Relationship between CT scan density and hematoma age on chronic subdural hematoma cases. Indonesian Journal of Neurosurgery 4(1): 11-16. D0I: 10.15562/ijn.v4i1.135

\section{INTRODUCTION}

Subdural hematoma (SDH) is a collection of blood below the inner layer of the dura mater but located outside the brain and arachnoid membrane. Subdural hematomas are the most common type of intracranial lesions due to trauma. Subdural hematomas can occur in one in three people with severe head injuries. ${ }^{1}$ SDH cases are increasingly being found with age. Study by Karibe, et al. (2011) stated that the prevalence of SDH reached 76.5 cases per 100,000 population with the age of 70-79 years and 127.1 cases per 100,000 population at the age above 80 years. ${ }^{2}$ An epidemiological study based in the United Kingdom, found that the annual incidence of SDH in children was estimated at 12.5 cases per 100,000 population with an age range of $0-2$ years and an estimated 24 cases per 100,000 in the age range of $0-1$ years. Most (57\%) of SDH cases that occur are caused by accidental injuries, other causes include: perinatal complications; meningitis; and other non-traumatic medical conditions. Spontaneous intracranial hypotension is also reported as a rare cause. ${ }^{3}$

Subdural hematomas are one of the most common clinical entities in neurosurgery practice and are a frequent consequence of trauma. In establishing the diagnosis, different clinical features may be found, varying from potentially lethal conditions such as acute subdural hematomas, to chronic hidden subdural hematoma (CSDH). Mild subdural hematomas can be found on computerized tomography (CT) scans and, in most cases, do not require special treatment. Spontaneous subdural hematomas (nontraumatic) are rarely diagnosed, such as, after hemorrhage aneurysms (associated with poor outcomes), other medical conditions (hematology, infectious diseases, lymphoid nodular metastases). ${ }^{4}$

The pathophysiology of subdural hematoma is currently widely studied, with heterogeneous characteristics that are time dependent. About $1 \%$ to $6 \%$ of 
untreated acute subdural hematomas will transform into chronic $\mathrm{SDH}^{5}$, on average 3 weeks after the trauma event. However, these two entities display significantly different manifestations and dynamic processes. Within hours or even days or weeks, the intrinsic characteristics of SDH can vary due to chronicity, reabsorption, or rebleeding in the type of chronic or subacute SDH. Computerized tomography scanning has been an important diagnostic tool. The current SDH imaging display is still used as a single criterion in different systematization. However, the current classification is still considered to be less correlated with clinical application. ${ }^{6}$ The terminology that is currently widely known (chronic, subacute, and acute division) is considered to be simple, lacking precision, and, ineffective in providing concise and clear information. This setting is still influenced by the patient's hematocrit level, clotting ability, or the possibility of rebleeding.

One type of classification, subacute subdural hematomas, is considered to be very subjective, because it includes different macroscopic appearance and different definitions of the relevant timings. In contrast to the other two types of subdural hematoma, the clinical characteristics of subacute subdural hematomas are rarely studied in studies and are not widely known. Subacute subdural hematomas and even clinically often overlap with the category of chronic subdural hematomas by some researchers. But the difference is that subacute subdural hematomas are mostly experienced by young patients and there are more progressive neurological defects. In addition, subacute subdural hematomas also leave problems in their administration. The density on the CT scan that has been used as a reference for action, still cannot provide clear boundaries. $^{7}$

Specific consensus is now needed, especially regarding the classification criteria that strengthen the surgical feasibility according to pathophysiological parameters and clinical correlations. A more standard approach to SDH gradation is needed, related to the governance of each type. Studies that explain the correlation of histopathological changes in hematoma and radiological contents of cases of subdural hematoma are still very limited. In this research, SDH histopathological changes are expected to be a reference and consideration in the next invasive therapeutic action. This study also aims to look for other approaches in determining the therapeutic actions in acute subdural hematomas by comparing the clinical, radiological, and histopathological profiles of blood deposits from subdural hematomas.

\section{METHODS}

This study is a clinical observational study with a cross sectional study approach. The study was carried out at Dr. Soetomo Academic General Hospital. The time of the study was carried out during February 2017 to February 2020. Patients who met the criteria were taken from a hematoma, and analysed at the Anatomy Pathology Laboratory of the Faculty of Medicine, Airlangga University.

The study population was subdural hematoma patients in the ER (emergency room) at Dr. Soetomo Academic General Hospital Surabaya, from January to December 2018, after that period the research process was completed. The study sample was patients with subdural hematoma at the ER, the period of January - December 2018 that meets the inclusion and exclusion criteria. The research sample was taken by consecutive sampling. Subjects were examined for head CT scan and retrieval.

The inclusion criteria of the study were: patients who came to the ER of our hospital with GCS 4 to 15 and had not received surgery with a head CT scan showed a picture of a subdural hematoma that was included in the indication criteria for surgery, and was willing to participate in the study which is stated with an informed consent form that has been signed by the patient or guardian concerned. Whereas the study exclusion criteria were: patients who received anticoagulant therapy, patients with CT images of the head within normal limits, patients with CT images of the head showing hematomas other than subdural, or images of subdural hematomas that were not indicative of surgery, and patients with haematological disease.

Primary data were obtained from all subdural hematoma patients with several characters (age, sex, GCS score, blood pressure, pulse per minute, respiratory rate per minute, body temperature). Blood pressure is measured using a digital manometer. Body temperature is measured using a thermometer. CT Scan was performed in the Radiology Department and the results were read by Radiologists. Hematoma blood samples was analysed microscopically and morphologically on Pathological Anatomy Department of our hospital and was analysed by pathologist.

\section{RESULTS}

\section{Patients Characteristics}

A total of 40 patient data met the criteria and were processed for further analysis. Based on the results of research data, as many as 22 patients with male sex and 18 patients with female sex. The mean age of patients as a whole was 47.94 ( \pm 29.45) years with an age range of 3 months to 93 years. According to sex, the mean age of patients was $55.95( \pm 27.05)$ years for males and 30.38 ( \pm 30.74 ) years. Patient characteristics are presented in Table 1.

\section{Blood Density Reading Values on CT Scan (Hounsfield Unit)}

Blood density values on CT scan images are taken from reading images processed by a computer in units of Hounsfield Unit (HU). HU data values were obtained from readings in the patient's medical record. From the analysis results it can be seen the average value of CT Scan blood reading density of Chronic SDH patients at Dr. Soetomo Academic General Hospital which was operated on in 2018-2019 was $31.30( \pm 11.47) \mathrm{HU}$ with the smallest value of 4.0 and the largest $54 \mathrm{HU}$. Data is presented in Table 2.

\section{Number of Days of Chronic SDH Patient Occurrence}

The day of the incident in Chronic SDH patients at Dr. Soetomo Academic General Hospital, who was operated in 2018-2019, was taken from medical records. The number of days of events is calculated from the day of events in the data history of the patient to the day of data collection. From the results of the analysis, the average day of events was $36 \pm 36.66$ days 
with the shortest occurrence day 12 days and the longest 150 days. Data is presented in Table 3.

\section{Statistical Analysis}

From Chronic SDH patient data at Dr. Soetomo Academic General Hospital, who was operated on in 2018-2019, performed a statistical analysis to find the correlation between the duration of the event and the CT Scan reading density. The results of the statistical analysis show that there is an influence between the CT Scan reading density and the day of the incident. The calculation results obtained a negative correlation $(\mathrm{r}=-0.814 ; \mathrm{p}<0.001)$ between the CT Scan density value and the day of occurrence in Chronic SDH patients. The longer the day the incident has a correlation with the CT Scan density reading value is getting lower. The results of the statistical analysis are presented in Table 4.

Table 1. Characteristics of data of patients with Chronic SDH operated on in Dr. Soetomo Academic General Hospital, Surabaya in 2018-2019

\begin{tabular}{cccc} 
& \multirow{2}{*}{ Sex } & Total & \multicolumn{2}{c}{ Age (Year) } \\
\cline { 3 - 4 } & & Mean & Standard Deviation \\
\hline Male & 22 & 55,95 & 27,05 \\
Female & 18 & 30,38 & 30,74 \\
Total & 40 & 47,94 & 29,45
\end{tabular}

Table 2. Characteristics of CT Scan density readings for Chronic SDH patients operated at Dr. Soetomo Academic General Hospital Surabaya in 2018-2019.

\begin{tabular}{ccccc} 
& Total & Mean & $\begin{array}{c}\text { Standard } \\
\text { Deviation }\end{array}$ & Min - Max \\
\hline CT Scan Density & 40 & 31,3 & 11,47 & $4-54$ \\
\hline
\end{tabular}

Table 3. Characteristics of day occurrence of Chronic SDH patients operated at Dr. Soetomo Academic General Hospital, Surabaya in 2018-2019

\begin{tabular}{lcccc}
\hline & Total & Mean & Standard Deviation & Min - Max \\
\hline $\begin{array}{l}\text { Number of } \\
\text { Days }\end{array}$ & 40 & 35 & 36,66 & $12-150$ \\
\hline
\end{tabular}

Table 4. Correlation of CT Scan density values with the day of occurrence of Chronic SDH patients operated at Dr. Soetomo Academic General Hospital, Surabaya in 2018-2019.

\begin{tabular}{lcccccc}
\multicolumn{1}{c}{ Model } & $\begin{array}{c}\text { Sum of } \\
\text { Squares }\end{array}$ & df & Mean Square & F & p & $\begin{array}{c}\text { Pearson } \\
\text { Correlation }\end{array}$ \\
\hline Regression & 34724,98 & 1 & 34724,98 & 74,60 & 0,001 & $-0,814$ \\
Residual & 17689,02 & 38 & 465,50 & & & \\
Total & 52414,00 & 39 & & & & \\
\hline
\end{tabular}

a. Dependent variable: Number of Days After Accident

b. Independent Variable: Housenfield Unit

Table 5. Calculation of HU coefficients and Day of Occurrence

\begin{tabular}{lcccc}
\multicolumn{1}{c}{ Variabel } & B & Std. Eror & $\mathbf{t}$ & $\mathbf{p}$ \\
\hline Number of Days & 116,415 & 10,025 & 11,613 & 0,001 \\
HU & $-2,601$ & 0,301 & $-8,637$ & 0,001 \\
\hline
\end{tabular}

It is determined that the dependent variable is the day of occurrence in units of days and the independent variable is the $\mathrm{HU}$ value of the CT Scan reading. The statistical calculation of the coefficient B0 for the dependent variable (day of occurrence) is 147.557 and the coefficient of B1 for the HU value from the CT Scan reading is -3.417 .

Statistical analysis shows negative correlations that can be translated into formulas to determine the day of occurrence / age of the hematoma as follows:

Age of Hematoma (Day) $=\mathrm{B} 0+\mathrm{B} 1(\mathrm{HU})$

$\mathrm{B} 0=116,415$

$\mathrm{B} 1=-2,601$

$\mathrm{HU}=\mathrm{HU}$ value from blood Region of Interest (ROI) Readings from CT Scan

Then the hematoma age calculation formula is obtained as follows:

\section{Hematoma Age (Days) $=$ $116,415-2,601(\mathrm{HU})$}

The graphic of the distribution of CT Scan density readings to the day of events is presented in the form of a Scatter Plot diagram. From the results of the distribution of graphs, we can see a nonlinear, strong negative correlation between the CT Scan density reading values and the day of the event. The diagram illustration is presented in Figure 1.

Histopathological examination of blood from the preparation of the operation results showed the presence of blood cells that confirm the results of chronic subdural hematoma surgery. Lysis of erythrocyte cells, PMN, lymphocytes, macrophages and fibrin. Lysis erythrocyte cells can be detected in all readings from the operation to confirm subdural bleeding.

PMN cells can be detected in 25 cases with $\mathrm{HU}$ values on positive $\mathrm{PMN}$ readings $(n=25)$ of $33.76 \pm 10.90$ with the smallest $\mathrm{HU}$ values 11 and the largest 54; while the $\mathrm{HU}$ value on the PMN reading was negative $(n=15)$ of $27.20 \pm 11.58$ with the smallest $\mathrm{HU}$ value 4 and the largest 42 .

Lymphocyte cells can be detected in 36 cases with $\mathrm{HU}$ values on positive 


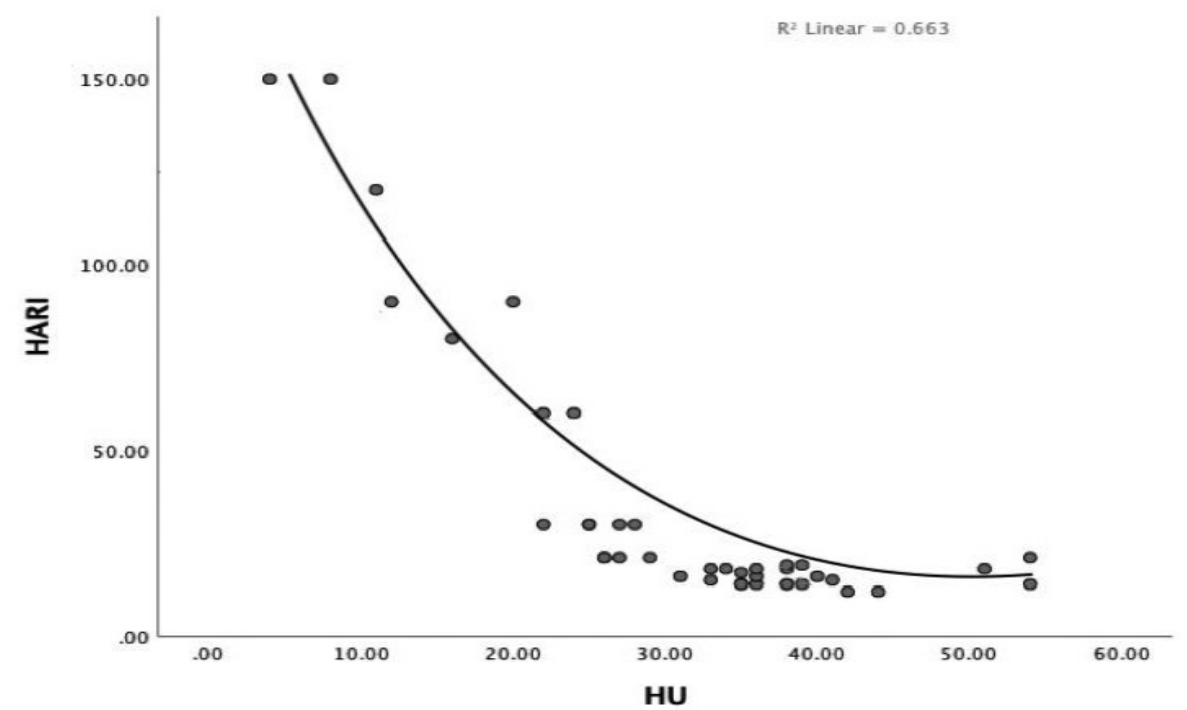

Figure 1. Scatter Plot of CT Scan (HU) density with day of occurrence

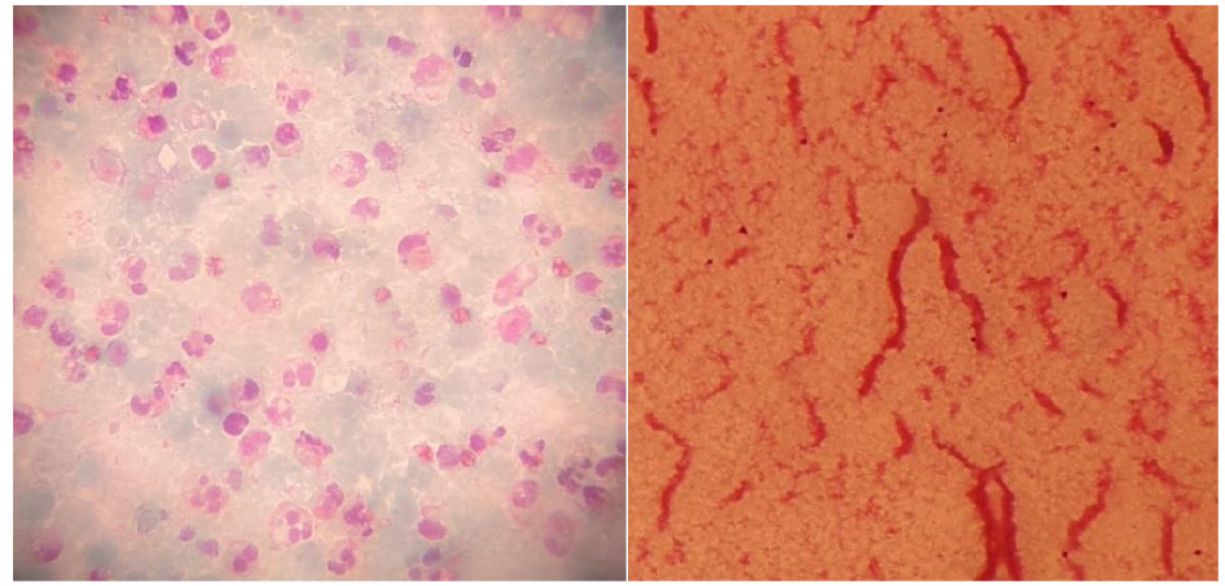

Figure 2. Histopathological features of chronic SDH. PMN cells, lymphocytes, and fibrin can be found in most chronic SDH examination preparations. lymphocyte readings $(\mathrm{n}=36)$ of $30.81 \pm$ 11.88 with the smallest $\mathrm{HU}$ value 4 and the largest 54; while the $\mathrm{HU}$ value on negative lymphocyte readings $(\mathrm{n}=4)$ was $35.75 \pm$ 5.85 with the smallest $\mathrm{HU}$ value 29 and the largest 42.

Macrophage cells can be detected in 25 cases with $\mathrm{HU}$ values on positive macrophage readings $(n=25)$ of $29.64 \pm$ 13.26 with the smallest 4 and biggest $\mathrm{HU}$ values; while the $\mathrm{HU}$ value on negative macrophage readings $(\mathrm{n}=15)$ was 34.07 \pm 7.22 with the smallest $\mathrm{HU}$ value 26 and the largest 51.

Fibrin can be detected in 33 cases with $\mathrm{HU}$ values on positive $\mathrm{PMN}$ readings $(\mathrm{n}=$ 33) of $31.45 \pm 11.89$ with the smallest $\mathrm{HU}$ value 4 and the largest 54; while the $\mathrm{HU}$ value on the negative PMN reading $(\mathrm{n}=$ 7) was $30.57 \pm 10.05$ with the smallest $\mathrm{HU}$ value 11 and the largest 41 .

\section{DISCUSSION}

\section{CT Scan Density with Event Day}

The appearance of a hematoma on a CT scan can be a predictor of postoperative recurrence. ${ }^{8}$ In clinical analysis, taking into account the risk factors associated with $\mathrm{CSDH}$ recurrence, the recurrence incidence in the high density or mixed group was significantly higher than the low density group or iso-density.

In this study, the overall age of the patients was $47.94( \pm 29.45)$ years with an age range of 3 months to 93 years.

Table 6. Table of cell reading results on histopathological examination compared with the value of blood CT Scan (HU) readings.

\begin{tabular}{|c|c|c|c|c|c|c|c|}
\hline \multirow[b]{2}{*}{ Cell } & \multirow[b]{2}{*}{$+/-$} & \multirow[b]{2}{*}{$\mathbf{N}$} & \multicolumn{4}{|c|}{ HU } & \multirow[b]{2}{*}{$\mathbf{p}$} \\
\hline & & & Mean & $\begin{array}{l}\text { Standard } \\
\text { Deviation }\end{array}$ & Minimum & Maximum & \\
\hline \multirow{2}{*}{ Lysis Erythrocytes } & + & 40 & 31,3 & 11,47 & 4 & 54 & \\
\hline & - & 0 & - & - & - & - & \\
\hline \multirow{2}{*}{ PMN } & + & 25 & 33,76 & 10,90 & 11 & 54 & \multirow{2}{*}{0,080} \\
\hline & - & 15 & 27,2 & 11,58 & 4 & 42 & \\
\hline \multirow{2}{*}{ Lymphocyte } & + & 36 & 30,81 & 11,88 & 4 & 54 & \multirow{2}{*}{0,421} \\
\hline & - & 4 & 35,75 & 5,85 & 29 & 42 & \\
\hline \multirow{2}{*}{ Macrophage } & + & 25 & 29,64 & 13,26 & 4 & 54 & \multirow{2}{*}{0,242} \\
\hline & - & 15 & 34,07 & 7,22 & 26 & 51 & \\
\hline \multirow{2}{*}{ Fibrin } & + & 33 & 31,45 & 11,89 & 4 & 54 & \multirow{2}{*}{0,856} \\
\hline & - & 7 & 30,57 & 10,05 & 11 & 41 & \\
\hline
\end{tabular}


According to sex, the mean age of patients was $40.24( \pm 29.13)$ years for males and $55.65( \pm 28.40)$ years. The results of the statistical analysis show that there is an influence between the CT Scan reading density and the day of the incident. The calculation results obtained negative nonlinear correlation $(\mathrm{r}=-0.676 ; \mathrm{p}<0.001)$ between the CT Scan density value and the day of occurrence in Chronic SDH patients. The longer the day the event has a nonlinear correlation with CT Scan readings the lower the density.

Blood density values on CT scan images are taken from reading images processed by a computer in units of Hounsfield Unit (HU). HU data values were obtained from readings in the patient's medical record. From the analysis results it can be seen the average value of CT Scan blood reading density of Chronic SDH patients at Dr. Soetomo Academic General Hospital which was operated on in 2018-2019 was $31.30( \pm 11.47) \mathrm{HU}$ with the smallest value of 4.0 and the largest $54 \mathrm{HU}$.

The day of the incident in Chronic SDH patients at Dr. Soetomo Academic General Hospital, who was operated on in 2018-2019, was taken from medical records. The number of days of events is calculated from the day of events in the data history of the patient to the day of data collection. From the results of the analysis, the average day of events was $36 \pm 36.66$ days with the shortest occurrence day 12 days and the longest 150 days. Statistical analysis showed that there was an influence between the CT Scan reading density and the day of the incident. The calculation results obtained a negative correlation ( $\mathrm{r}$ $=-0.814 ; \mathrm{p}<0.001)$ between the CT Scan density value and the day of occurrence in Chronic SDH patients. The longer the day the incident has a correlation with the CT Scan density reading value is getting lower. A different matter was found by Sieswerda-Hoogendoorn et al. where a systematic review of 17 studies examining the relationship between CT Scan density and SDH age had overlapping values. Similarly, 5 studies analyzed for similar examinations using MRI. According to Sieswerda-Hoogendoorn et al. it is difficult to determine the age of bleeding in SDH cases based on radiological examination. However, their research is limited by variations in data with a wide range and small scale of research. ${ }^{9}$ This is what underlies the importance of histopathological examination to confirm the age of SDH.

\section{Histological Overview of SDH}

Red blood cell lysis was observed in part starting at PTI (post traumatic interval) 7 hours. Most cases with complete lysis of red blood cells have been found to have PTI more than 96 hours. PMN is observed from minimum PTI to maximum PTI. There are no cases where neutrophils are very rare or absent. Macrophages, red blood cells containing macrophages, macrophages containing haemosiderin, fibroblasts, collagen fibers, initial membrane formation, endothelial proliferation were first observed in PTI 17.3 hours, 24.5 hours, 80 hours, 89.5 hours, 110, 5 hours, 114.5 hours, and 123 hours respectively. ${ }^{10}$

In this study, the histology generally did not represent chronic SDH readings specifically. Chronic SDH starts as a thin blood clot between the dura and the arachnoid that was not originally attached to the dura. Fibroblasts, which grow from the dura into lumps, arrange it. Within 5-6 days, fibroblast growth causes blood clots to be loosely attached to the dura. Within 10-20 days, a loose fibrous membrane is formed between the dura and the clot (outer membrane). Fibrous tissue then grows around the edge of the hematoma and along the inner surface (inner membrane), surrounds it completely. Maturation of connective tissue, after a few weeks or months, causes the formation of sacs with fibrous walls (chronic SDH). The blood in these sacs is absorbed to varying degrees, and the cavity contains clear or haemorrhagic fluid and loose and vascular connective tissue. Rupture of blood vessels can cause recurrent bleeding in the sac. Fluid can also leak into the cavity of immature capillaries. If large amounts of cerebrospinal fluid (CSS) enter the subdural space during a traumatic event, then CSS will bleed, and no clotting or organization occurs. Histological examination of the sac is very helpful in estimating the duration of $\mathrm{SDH} .{ }^{11}$

\section{Radiological features in SDH}

In comparison the use of CT Scan and MRI itself in the case of SDH has different interests. CT scan remains the first choice in early SDH cases. The availability, speed, and sensitivity of the examination results in the identification of intracranial haemorrhage are the basis of these considerations. ${ }^{12}$ However CT scan has disadvantages compared to MRI where CT scan is less able to detect changes that occur in the cortical structure of the brain, especially changes with small lesions. ${ }^{12,13}$ Atkinson reported in a case study in which MRI examination was able to estimate the evolution of SDH that occurred based on a picture of pacimeningeal thickening (dura-arachnoid) after gadolinium administration. ${ }^{14}$ Lee et al. shows that the prognosis and prediction of the risk of burrhole action and its operating outcomes in the DiffusionWeighted MRI examination can be estimated from the signal reading results with mixed-intensity. Signal readings with heterogeneous intensity have worse output values when compared to signals which are heterogeneous. ${ }^{15}$ Although both modalities are able to diagnose SDH cases, MRI is superior in the sensitivity of diagnosing bilateral chronic SDH cases, multiple loculation, membrane features, haemolysis, and large capsule measurements. ${ }^{16}$ Both modalities have the potential for shared use in completing the diagnosis and understanding of SDH cases.

\section{Relationship of CT Scan Density with SDH Histological Features}

In a study conducted by Benson et al., A relationship was found between attenuation of CT Scan density and the composition of the histological picture of the blood. The results show that there is a relationship between permeability of blood clots and the relative density of red blood cells and fibrin in blood clots. An increase in absolute arterial attenuation of HU10 HU in CTA compared to NCCT was associated with a relatively higher $\mathrm{RBC}$ density and lower fibrin density in the collected clot. ${ }^{17}$

Data shown in another study by Borggrefe et al. showed that blood clot fibrin content was associated with low 
density on unreinforced CT and increased specific density after increased contrast caused by marked contrast absorption in clumps that were significantly higher than clots which is rich in red blood cells. ${ }^{18}$ Fibrin proteins appear to have a higher affinity for contrast media than cell-rich clumps, as previously assumed in our hypothesis. The relative fibrin content in the clot analogue is associated with lower density on CT which is not enhanced and the results show a positive linear correlation with increased contrast. In addition, there is a positive relationship between RBC content and clot density in CT scans that have not been enhanced, which have been demonstrated previously and can be explained by the haematocrit level in the clot. ${ }^{19}$ The results in this study differed from our study in which this study did not explore the relationship of CT Scan density to each cell found in the histological picture due to the lack of quantitative data obtained in hematoma cytology examination. More in-depth research is needed to learn more about this. The different components of each cell in the hematoma have the potential to provide a different HU picture. ${ }^{18}$

Most previous investigations of clot permeability based on CT imaging have focused on the relationship between clot imaging characteristics and response to treatment or clinical outcomes. The correlation found between these variables has been linked to several factors: clumps that appear on CT with a relatively inhomogeneous picture, allow the passage of residual arterial flow and maintain at least some oxygenation to the downstream tissue. Thrombolysis IV as a translucent blob penetrating may be a reasonable biomarker of tPA drug administration to the clot itself. For endovascular recanalization therapy, most studies examining the relationship between perviousness and thrombectomy results have concluded that patients with see-through clots are more likely to be successfully repeated and will have better results. However this idea has been refuted in at least one subsequent study. ${ }^{20,21}$

\section{CONCLUSIONS}

There's a negative correlation between the number of incident days with the CT Scan density where the longer the event day the lower the CT Scan density value

\section{ACKNOWLEDGMENTS}

Non declared.

\section{DECLARATIONS}

\section{Funding}

No specific funding.

\section{Conflict of interest}

Authors have no conflict of interest.

\section{Ethical approval}

This study had been approved by Ethical Committee of Dr. Soetomo Academic General Hospital

\section{Authors' contributions}

Authors took part in design of the study and contributed to data collection. ZRA did literature review and drafted the manuscript. AT, SAU, DF made critical revisions to the mauscript and all agree to accept equal responsibility for accuracy of the contents of this article.

\section{REFERENCES}

1. Tallon, J. M., Ackroyd-Stolarz, S., Karim, S. A. \& Clarke, D. B. The epidemiology of surgically treated acute subdural and epidural hematomas in patients with head injuries: A populationbased study. Can. J. Surg. 51, 339-345 (2008).

2. Karibe, H., Kameyama, M., Kawase, M. \& Tominaga, T. Epidemiology of chronic subdural hematoma. No Shinkei Geka, Neurol. Surg. 39, 1149-1153 (2011).

3. Hobbs, C., Childs, A. M., Wynne, J., Livingston, J. \& Seal, A. Subdural haematoma and effusion in infancy: An epidemiological study. Arch. Dis. Child. 90, 952-955 (2005).

4. Louhab, N. et al. Misdiagnosed spontaneous intracranial hypotension complicated by subdural hematoma following lumbar puncture. Int. J. Gen. Med. 7, 71-73 (2014).

5. Vezina, G. Assessment of the nature and age of subdural collections in nonaccidental head injury with CT and MRI. Pediatr. Radiol. 39, 586-590 (2009).

6. Postema, F. A. M., Sieswerda-Hoogendoorn, T., Majoie, C. B. L. M. \& Van Rijn, R. R. Age determination of subdural hematomas: Survey among radiologists. Emerg. Radiol. 21, 349-358 (2014).

7. Rovlias, A., Theodoropoulos, S. \& Papoutsakis, D. Chronic subdural hematoma: Surgical management and outcome in 986 cases: A classification and regression tree approach. Surg. Neurol. Int. 6, (2015).

8. Jeong, S. Il, Kim, S. O., Won, Y. S., Kwon, Y. J. \& Choi, C. S. Clinical Analysis of Risk Factors for Recurrence in Patients with Chronic Subdural Hematoma Undergoing Burr Hole Trephination. Korean J. Neurotrauma 10, 15 (2014).

9. Sieswerda-Hoogendoorn, T., Postema, F. A. M., Verbaan, D., Majoie, C. B. \& Van Rijn, R. R. Age determination of subdural hematomas with CT and MRI: A systematic review. Eur. J. Radiol. 83, 1257-1268 (2014).

10. Rao, M. G., Singh, D., Vashista, R. K. \& Sharma, S. K. Dating of acute and subacute subdural haemorrhage: A histo-pathological study. J. Clin. Diagnostic Res. 10, HC01-HC07 (2016).

11. Bokka, S. \& Trivedi, A. Histopathological study of the outer membrane of the dura mater in chronic sub dural hematoma: Its clinical and radiological correlation. Asian J. Neurosurg. 11, 34 (2016).

12. Hedlund, G. L. \& Frasier, L. D. Neuroimaging of abusive head trauma. Forensic Sci. Med. Pathol. 5, 280-290 (2009).

13. Foerster, B. R. et al. Neuroimaging Evaluation of Non-accidental Head Trauma with Correlation to Clinical Outcomes: A Review of 57 Cases. J. Pediatr. 154, 573-577 (2009).

14. Atkinson, J. L. D., Lane, J. I. \& Aksamit, A. J. MRI depiction of chronic intradural (subdural) hematoma in evolution. J. Magn. Reson. Imaging 17, 484-486 (2003).

15. Lee, S. H. et al. The potential of diffusionweighted magnetic resonance imaging for predicting the outcomes of chronic subdural hematomas. J. Korean Neurosurg. Soc. 61, 97104 (2018).

16. Yadav, Y. et al. Role of endoscopic third ventriculostomy in tuberculous meningitis with hydrocephalus. Asian J. Neurosurg. 11, 325 (2016).

17. Benson, J. C. et al. Clot permeability and histopathology: Is a clot's perviousness on CT imaging correlated with its histologic composition? J. Neurointerv. Surg. 12, 38-42 (2020).

18. Borggrefe, J. et al. Differentiation of Clot Composition Using Conventional and Dual-Energy Computed Tomography. Clin. Neuroradiol. 28, 515-522 (2018).

19. Kirchhof, K., Welzel, T., Mecke, C., Zoubaa, S. \& Sartor, K. Differentiation of white, mixed, and red thrombi: Value of CT in estimation of the prognosis of thrombolysis - Phantom study. Radiology 228, 126-130 (2003).

20. Niesten, J. M. et al. Histopathologic composition of cerebral thrombi of acute stroke patients is correlated with stroke subtype and thrombus attenuation. PLoS One 9, 12-14 (2014).

21. Santos, E. M. M. et al. Permeable Thrombi Are Associated with Higher Intravenous Recombinant Tissue-Type Plasminogen Activator Treatment Success in Patients with Acute Ischemic Stroke. Stroke 47, 2058-2065 (2016).

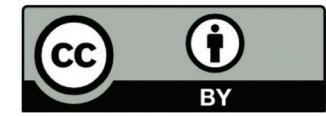

This work is licensed under a Creative Commons Attribution 